\title{
Comportamento da remoção de matéria organica (DQO) e nitrogênio amoniacal em um reator de leito estruturado operado sob aeração intermitente
}

\author{
Matter of removal of organic (COD) behavior and nitrogen ammoniacal in bed reactor of \\ structured operated under intermittent aeration
}

\author{
C. Z. Correa ${ }^{1 *}$; K.V. M. C. Prates ${ }^{1}$; C. S. Azevedo ${ }^{2}$; D. D. Lopes ${ }^{3}$; E. A. \\ Rodrigues $^{4}$; G. Peterlini ${ }^{4}$; A. C. Barana ${ }^{5}$
}

${ }^{1}$ Programa de Pós graduação em Engenharia Ambiental, Universidade Tecnológica Federal do Paraná, CEP 86036370, Londrina-PR, Brasil, ' ${ }^{2}$ Graduação em Engenharia Civil, Universidade Estadual de Londrina, CEP 86051-991, Londrina-PR, Brasil; ${ }^{3}$ Centro de Tecnológia e Urbanismo, Universidade Estadual de Londrina, CEP 86051-991, Londrina-PR, Brasil ; ${ }^{4}$ Graduação em Engenharia Ambiental, Universidade Tecnológica Federal do Paraná, CEP 86036-370, Londrina-PR, Brasil; ${ }^{5}$ Programa de Pós graduação em Engenharia de Alimentos, Universidade Estadual de Ponta Grossa, CEP 84030-900, Ponta Grossa- PR, Brasil

*camila.z.correa@gmail.com

(Recebido em 11 de agosto de 2015; aceito em 23 de fevereiro de 2016)

\begin{abstract}
O objetivo deste trabalho foi avaliar o comportamento da remoção de matéria orgânica (Demanda Química de Oxigênio - DQO) e nitrogênio ( $\mathrm{N}$-amoniacal $-\mathrm{NH}_{4}{ }^{+}$) em um reator de leito estruturado e fluxo contínuo, operado com aeração intermitente (AI). O reator utilizado possuía volume útil de 9,4L, e foi preenchido com estruturas verticais de espuma de poliuretano. $\mathrm{O}$ sistema foi operado durante 38 dias sob aeração intermitente ( $4 \mathrm{~h}$ aerado (AE) e $2 \mathrm{~h}$ não aerado (AN)) com taxa de recirculação de 3 vezes a vazão de entrada. O tempo de detenção hidráulico (TDH) foi de $16 \mathrm{~h}$. Durante a operação do reator foram realizadas análises para determinação do $\mathrm{pH}$, alcalinidade, temperatura, $\mathrm{DQO}, \mathrm{N}$-amoniacal $\left(\mathrm{NH}_{4}{ }^{+}\right)$, nitrito $\left(\mathrm{N}^{-} \mathrm{NO}_{2}{ }^{-}\right)$, nitrato $\left(\mathrm{N}^{-\mathrm{NO}_{3}}{ }^{-}\right.$), Nitrogênio Kjeldhl Total (NTK) e Sólidos em Suspensão Voláteis (SSV). No $38^{0}$ dia foram realizadas análises durante 3 ciclos de AI, totalizando $18 \mathrm{~h}$ de obtenção de dados, sendo realizadas coletas de efluente de $1 \mathrm{em} 1 \mathrm{~h}$, para determinar o perfil do consumo de $\mathrm{DQO}$ e $\mathrm{NH}_{4}{ }^{+}$. O reator atingiu eficiência média de $88 \%$ em termos de remoção de $\mathrm{DQO}_{\mathrm{T}}$ e $72 \%$ de remoção de NTK, durante os 38 dias de operação. Em relação ao perfil obtiveram-se remoções médias de $\mathrm{DQO}_{\mathrm{T}}$ e $\mathrm{N}-\mathrm{NH}_{4}{ }^{+}$na fase $\mathrm{AE}$ de $92 \pm 1 \%$ e $49 \pm 8 \%$, respectivamente, e na fase $\mathrm{AN}$ foram obtidos percentuais médios de remoção de $\mathrm{DQO}_{\mathrm{T}}$ de $90 \pm 4 \%$ e $\mathrm{N}-\mathrm{NH}_{4}{ }^{+}$de $46 \pm 6 \%$. Dos resultados foi possível concluir que a AI não afetou de forma significativa o percentual de remoção de $\mathrm{DQO}_{\mathrm{T}}$ e $\mathrm{N}-\mathrm{NH}_{4}{ }^{+}$no reator.
\end{abstract}

Palavras-chave: Aeração intermitente, Reator de leito estruturado, Espuma de poliuretano.

The arms of this study was to evaluate the behavior of the removal of organic matter (Chemical Oxygen Demand - COD) and nitrogen (N-ammonia $-\mathrm{NH}_{4}^{+}$) in a reactor of structured and continuous stream bed, operated with intermittent aeration (AI). The reactor used had useful volume of $9.4 \mathrm{~L}$ and vertical structures was filled with polyurethane foam. The system was operated for 38 days under intermittent aeration (4h aerated (EA) and non-aerated $2 \mathrm{~h}$ (NA)) with recirculation rate of 3 times the inlet flow. The hydraulic retention time (HRT) worked was $16 \mathrm{~h}$. During reactor operation analyzes were performed for determination of $\mathrm{pH}$, alkalinity, temperature, $\mathrm{COD}, \mathrm{N}$-ammonia $\left(\mathrm{NH}_{4}^{++)}\right.$, nitrite $\left(\mathrm{N}^{-\mathrm{NO}_{2}}{ }^{-}\right)$, nitrate $(\mathrm{N}-$ $\mathrm{NO}_{3}{ }^{-}$), Kjeldhl Total Nitrogen (NTK) and volatile suspended solids (VSS ). The analyzes were performed $38^{0}$ days for 3 cycles IA, a total of 18 hours of obtaining data of first effluent collections being made at 1 $\mathrm{hr}$ to determine the consumption pattern of $\mathrm{COD}$ and $\mathrm{N}-\mathrm{NH}_{4}{ }^{+}$. The reactor reached an average efficiency of $88 \%$ in terms of removing DQO $_{\text {T }}$ and $72 \%$ removal of NTK cells during the 38 days of operation. From the results obtained in the 18 hour analysis found average removal of $\mathrm{N}^{-N_{4}}{ }^{+}$and $\mathrm{DQO}_{\mathrm{T}}$, the EA phase of $92 \% \pm 1$ and $49 \pm 8 \% \mathrm{AN}$ and the phase $490 \pm 46 \%$ and $\pm 6 \%$ respectively. From the results it was concluded that the AI did not affect significantly the $\mathrm{DQO}_{\mathrm{T}}$ removal percentage and ammonia- $\mathrm{N}$ in the reactor.

Keywords: Intermittent aeration, Reactor structured bed, Polyurethane foam.

\section{INTRODUÇÃO}

Entre os principais contaminantes presentes nos esgotos sanitários destacam-se os sólidos em suspensão, compostos orgânicos (proteínas: 40 a 60\%; carboidratos: 25 a 50\%; e óleos e graxas: 
10\%), nutrientes (nitrogênio e fósforo), metais, sólidos dissolvidos inorgânicos, sólidos grosseiros, compostos não biodegradáveis, organismos patogênicos e, ocasionalmente, contaminantes tóxicos decorrentes de atividades industriais e/ou acidentais. Em geral, a remoção dos contaminantes, principalmente matéria orgânica e nitrogênio, é realizada por meio de sistemas de tratamento biológico. A remoção da matéria orgânica pode ser alcançada utilizando tanto o processo anaeróbio como o aeróbio ou, ainda, pela combinação dos dois.

No caso dos compostos nitrogenados, para sua efetiva remoção, é necessário a alternância dos processos, uma vez que convencionalmente a remoção destes compostos acontece por meio de dois processos sucessivos: a nitrificação, que é a conversão de amônia em nitrato e a desnitrificação, conhecido como sendo a redução do nitrato a nitrogênio gasoso, sendo este liberado na atmosfera sem causar danos ambientais [1]. O processo de nitrificação convencional, normalmente, é realizado por bactérias autotróficas aeróbias, e o processo de desnitrificação por bactérias heterotróficas facultativas que na ausência ou em baixas concentrações de Oxigênio

Dissolvido (OD) utilizam nitrato como aceptor final de elétrons.

Cada vez mais vem sendo desenvolvidas pesquisas que visam a nitrificação e a desnitrificação simultânea (NDS), em um mesmo reator, utilizando para tal sistemas de aeração intermitente AI $[2,3,4,5,6]$. O processo de NDS apresenta algumas vantagens como a redução do consumo de energia $[7,8]$ e a manutenção do $\mathrm{pH}$ próximo a neutralidade pois parte da alcalinidade consumida na nitrificação é restituída ao meio no processo de desnitrificação, resultando em menor demanda de alcalinidade externa [9] e economia de carbono orgânico, cujo aporte necessário pode ser reduzido em cerca de 22 a $44 \%$ [10].

Devido às vantagens relacionadas à utilização da NDS e aos sistemas operados com aeração intermitente, o presente trabalho teve como principal objetivo avaliar o efeito da aeração intermitente na remoção de matéria orgânica (Demanda Química de Oxigênio - DQO) e nitrogênio em um reator de leito estruturado.

\section{MATERIAL E MÉTODOS}

\subsection{INSTALAÇÃO EXPERIMENTAL E PERÍODO OPERACIONAL}

O reator foi alimentado com esgoto sanitário proveniente da Estação de Tratamento de Esgoto (ETE - norte) - SANEPAR/Londrina - PR, coletado após o tratamento preliminar (gradeamento e caixa de areia).

A instalação experimental utilizada, em escala de bancada, foi composta por um reator de leito estruturado com fluxo contínuo, com volume total de $13 \mathrm{~L}$ e volume útil de 9,4 L, diâmetro externo de $22 \mathrm{~cm}$, diâmetro interno de $16 \mathrm{~cm}$ e altura de $82 \mathrm{~cm}$. Como material suporte foram usadas estruturas cilíndricas de espuma de poliuretano com aproximadamente $3 \mathrm{~cm}$ de diâmetro e $65 \mathrm{~cm}$ de altura, totalizando 13 estruturas. Para a fixação das estruturas cilíndricas verticalmente no reator foram utilizadas hastes de PVC encaixadas nas extremidades do reator (Figura 1).

O reator foi alimentado com esgoto sanitário através de uma bomba magnética (ProMinent modelo GALA). Para a aeração foram utilizadas 3 bombas de aquário (Marca BIG ALFA A230), ligadas a pedras porosas que forneciam juntas uma vazão de ar de aproximadamente 5,0 L/min. Durante toda sua operação o efluente nitrificado foi recirculado a uma taxa de três vezes a vazão de entrada $\left(\mathrm{Q}_{\mathrm{Re}}=3 \mathrm{Q}\right)$. A recirculação foi realizada por meio de bomba da mesma marca e modelo da usada na alimentação. 


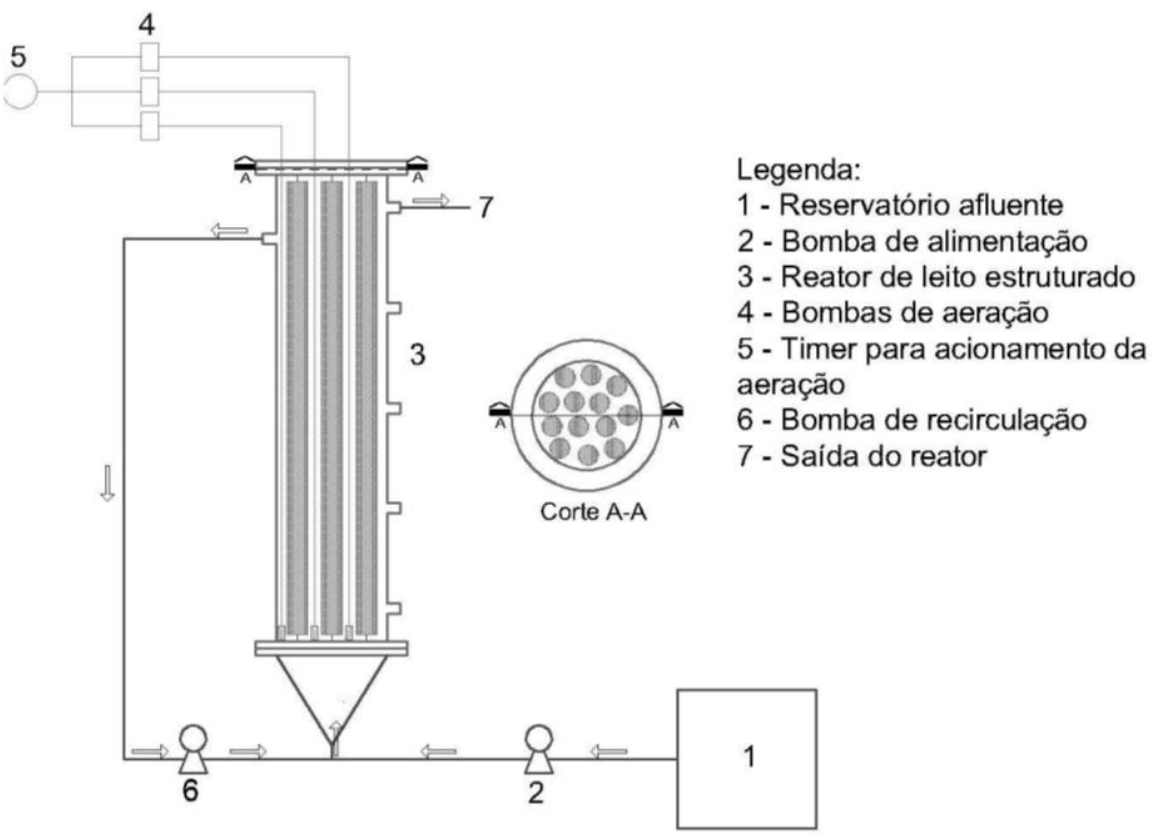

Figura 1: Representação esquemática do sistema de tratamento utilizado.

Para dar partida no reator foi utilizado um inóculo obtido por meio da aeração continua de esgoto sanitário, durante 20 dias em um reator em bateladas de $3 \mathrm{~L}$. Após este período foi constatada a atividade dos organismos nitrificantes e o lodo formado foi colocado, por um período de $24 \mathrm{~h}$, em contato com as espumas de poliuretano utilizadas como meio suporte. Transcorrido este tempo, as estruturas cilíndricas foram instaladas no reator que foi operado sob aeração e fluxo contínuo com TDH de $58 \mathrm{~h}$ para favorecer a adaptação e fixação da biomassa. Após 33 dias foi alterado o TDH para 16 horas e instalado o sistema de aeração intermitente. $O$ reator foi operado por 38 dias com este TDH e ciclos de aeração (AE) e não aeração (AN) de 4h/2h, respectivamente.

\subsection{MONITORAMENTO DOS PARÂMETROS FÍSICO-QUÍMICOS DURANTE O PERÍODO OPERACIONAL DO REATOR}

O monitoramento do sistema (análises físico-químicas do afluente e efluente) foi realizado seguindo os procedimentos descritos em APHA (2015)[11]. Foram realizadas análises 3 vezes por semana para determinação de $\mathrm{pH}(4500-\mathrm{H} \mathrm{B})$; alcalinidade total (2320 B); temperatura (2550 $\mathrm{B})$; Demanda Química de Oxigênio (DQO - $5220 \mathrm{D})$ total $\left(\mathrm{DQO}_{\mathrm{T}}\right)$ e filtrada $\left(\mathrm{DQO}_{\mathrm{F}}\right)$; Namoniacal- $\mathrm{NH}_{4}{ }^{+}\left(4500-\mathrm{NH}_{3} \mathrm{~B} / \mathrm{C}\right)$; nitrito- $\mathrm{N}^{-\mathrm{NO}_{2}}{ }^{-}\left(4500-\mathrm{NO}_{2} \mathrm{~B}\right)$; nitrato- $\mathrm{N}-\mathrm{NO}_{3}{ }^{-}\left(4500-\mathrm{NO}_{2} \mathrm{~B}\right)$. Uma vez por semana foram realizadas análises para determinação da concentração de Sólidos em Suspensão Voláteis - SSV (2540 E) e Nitrogênio Total Kjeldhl - NTK (4500 - Norg/4500-NH 3 C - Kjeldhl).

\subsection{PERFIL DE REMOÇÃO DE MATÉRIA ORGÂNICA E NITROGÊNIO DURANTE 3 CICLOS DE AI}

A fim de determinar a influência da aeração intermitente no consumo de matéria orgânica $\left(\mathrm{DQO}_{\mathrm{T}}\right)$ e oxidação de nitrogênio no reator foi realizado no $38^{\circ}$ dia de operação um ensaio que abrangeu 3 ciclos de AE e 3 ciclos de AN, totalizando 18 horas de monitoramento. As amostras foram coletadas em intervalos de $1 \mathrm{em} 1$ hora para determinação de $\mathrm{pH}$, alcalinidade $\left(\mathrm{mgCaCO}_{3} / \mathrm{L}\right), \mathrm{N}^{-\mathrm{NH}_{4}}{ }^{+}(\mathrm{mg} / \mathrm{L}), \mathrm{N}_{-} \mathrm{NO}_{2}{ }^{-}(\mathrm{mg} / \mathrm{L}), \mathrm{N}^{-\mathrm{NO}_{3}}{ }^{-}(\mathrm{mg} / \mathrm{L})$ e $\mathrm{DQO}_{\mathrm{T}}(\mathrm{mg} / \mathrm{L})$. Para determinação da concentração de OD no reator foi utilizado um Oxímetro (Modelo: HQ40d), com coleta de dados de OD de 5 em 5 minutos. 


\subsection{ANÁLISE ESTATÍSTICA}

A análise estatística dos resultados de $\mathrm{DQO}_{\mathrm{T}}, \mathrm{N}^{-\mathrm{NH}_{4}}{ }^{+}, \mathrm{NO}_{2}{ }^{-}$e $\mathrm{NO}_{3}{ }^{-}$obtidas no perfil (fase AE e AN) foram realizados no software livre BioEstat 5.0, aplicando o Teste $t$ aos dados amostrais, para duas amostras independentes, com um nível de decisão $\alpha=0,05$, para verificar se houve diferença nas fases analisadas entre estes parâmetros $\left(\mathrm{DQO}_{\mathrm{T}}, \mathrm{N}_{-} \mathrm{NH}_{4}{ }^{+}, \mathrm{NO}_{2}{ }^{-} \mathrm{e} \mathrm{NO}_{3}{ }^{-}\right)$.

\section{RESULTADOS E DISCUSSÃO}

\subsection{PARÂMETROS FÍSICO-QUÍMICOS DURANTE O PERÍODO OPERACIONAL DO REATOR}

Na Tabela 1 são apresentados os resultados de $\mathrm{pH}$, alcalinidade, temperatura, Demanda Química de Oxigênio Total $\left(\mathrm{DQO}_{\mathrm{T}}\right)$ e Filtrada $\left(\mathrm{DQO}_{\mathrm{F}}\right), \mathrm{SSV}$ afluente e efluente, a eficiência média de remoção de matéria orgânica e de sólidos e a carga orgânica volumétrica aplicada e removida durante os 38 dias de operação do reator.

Tabela 1: Média e desvio padrão de pH, alcalinidade total, temperatura, Demanda Química de Oxigênio Total $\left(D Q O_{T}\right)$ e Filtrada $\left(D Q O_{F}\right)$, SSV, porcentagem de remoção de DQO e SSV e carga orgânica volumétrica aplicada e removida.

\begin{tabular}{|c|c|c|c|}
\hline Parâmetros & Afluente & Efluente & \% Remoção \\
\hline pH & $8,0 \pm 0,6$ & $7,3 \pm 1,2$ & - \\
\hline Alcalinidade Total $\left(\mathrm{mgCaCO}_{3} / \mathrm{L}\right)$ & $290 \pm 86$ & $134 \pm 131$ & - \\
\hline Temperatura $\left({ }^{0} \mathrm{C}\right)$ & $28 \pm 2$ & $27,6 \pm 2$ & - \\
\hline $\mathrm{DQO}_{\mathrm{T}}(\mathrm{mg} / \mathrm{L})$ & $367 \pm 152$ & $42 \pm 17$ & $88 \pm 4$ \\
\hline $\mathrm{DQO}_{\mathrm{F}}(\mathrm{mg} / \mathrm{L})$ & $221 \pm 55$ & $40 \pm 17$ & $78 \pm 9$ \\
\hline $\operatorname{SSV}(\mathrm{mg} / \mathrm{L})$ & $252 \pm 242$ & $23 \pm 3$ & $89 \pm 1$ \\
\hline $\begin{array}{c}\text { Carga Orgânica Volumétrica aplicada } \\
\left(\mathrm{Kg.DQO} / \mathrm{m}^{3} . \mathrm{d}\right)\end{array}$ & & $0,51 \pm 0,23$ & \\
\hline $\begin{array}{l}\text { Carga Orgânica Volumétrica } \\
\text { removida }\left(\mathrm{Kg.DQO} / \mathrm{m}^{3} . \mathrm{d}\right)\end{array}$ & & $0,28 \pm 0,14$ & \\
\hline
\end{tabular}

Os resultados de $\mathrm{pH}$, alcalinidade, temperatura, DQO e SSV do esgoto sanitário afluente encontram-se dentro da faixa típica de valores observados no Brasil [12, 14]. O valor médio de DQO $_{\text {T }}$ efluente foi de $42 \pm 17 \mathrm{mg} / \mathrm{L}$ e de $\mathrm{DQO}_{\mathrm{F}}$ de $40 \pm 17 \mathrm{mg} / \mathrm{L}$, atingindo uma eficiência de remoção de matéria orgânica média de $88 \pm 4 \%$ de $\mathrm{DQO}_{\mathrm{T}}$ e $78 \pm 9 \%$ de $\mathrm{DQO}_{\mathrm{F}}$ ao final do período operacional do reator.

O comportamento da remoção de matéria orgânica no reator também foi avaliado por meio dos SSV, obtendo-se um desempenho do reator para este parâmetro de $88 \pm 1 \%$. Apura-se destes valores que o reator de leito estruturado obteve um bom desempenho em termos de remoção de matéria orgânica, apresentando baixa perda de sólidos; o que torna este sistema vantajoso quando comparado a sistemas que possuem apenas biomassa em suspensão, pois não há necessidade de decantadores após o tratamento para a separação da biomassa do efluente.

Barana et al. (2013) [14] utilizando um reator de leito estruturado com aeração intermitente (1h aeração/2 h não aeração) e TDH de $24 \mathrm{~h}$ para tratar um efluente com concentração de $\mathrm{DQO}_{\mathrm{T}}$ de $418 \mathrm{mg} / \mathrm{L}$ obtiveram um percentual de remoção de matéria orgânica de $95 \%$. Moura et al. (2012)[8] tratando esgoto sanitário sintético com concentração de $\mathrm{DQO}_{\mathrm{T}}$ de $364 \mathrm{mg} / \mathrm{L}$, também em um reator de leito estruturado com fluxo contínuo, aeração intermitente ( $2 \mathrm{~h}$ aeração/1 h não aeração), com TDH de 12 h, obtiveram eficiência de remoção de matéria orgânica de $89 \%$. Pelos resultados apresentados, constata-se que o reator de leito estruturado com aeração intermitente e fluxo contínuo remove percentuais consideravelmente elevados de matéria orgânica, sendo estes próximos ao percentual de remoção obtida nesta pesquisa.

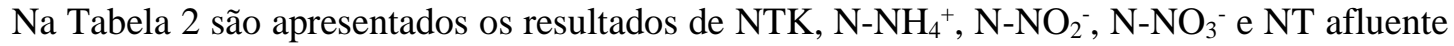
e efluente, juntamente com as eficiências calculadas e a carga nitrogenada aplicada e removida. A remoção média de NTK foi de $72 \pm 6 \%$, com concentração média efluente deste de $28 \pm 24 \mathrm{mg} / \mathrm{L}$. 
O percentual de remoção de $\mathrm{N}$-amoniacal foi de $70 \pm 21 \%$, com concentração média efluente de $21 \pm 16 \mathrm{mg} / \mathrm{L}$. A relação $\mathrm{N}$-amoniacal/NTK afluente ao reator foi de $0,6 \pm 0,1$ e efluente de $0,5 \pm 0,3$. A partir dos valores de $\mathrm{N}_{-} \mathrm{NO}_{2}{ }^{-}$efluente, em média $0,4 \pm 0,3 \mathrm{mg} / \mathrm{L}$, averígua-se que o processo de nitrificação biológica ocorreu até o último estágio de oxidação, com concentração média efluente de $\mathrm{N}^{-\mathrm{NO}_{3}}{ }^{-}$de $13 \pm 10 \mathrm{mg} / \mathrm{L}$, não sendo observado acúmulo de nitrito.

Tabela 2: Média e desvio padrão de $\mathrm{NTK}, \mathrm{N}-\mathrm{NH}_{4}{ }^{+}, \mathrm{N}-\mathrm{NO}_{2}{ }^{-}, \mathrm{N}-\mathrm{NO}_{3}{ }^{-}$, percentual de remoção de $\mathrm{N}$ amoniacal e carga nitrogenada aplicada e removida.

\begin{tabular}{|c|c|c|c|}
\hline Parâmetros & Afluente & Efluente & \% Remoção \\
\hline NTK (mg/L) & $86 \pm 51$ & $28 \pm 24$ & $72 \pm 6$ \\
\hline $\mathrm{N}-\mathrm{NH}_{4}{ }^{+}(\mathrm{mg} / \mathrm{L})$ & $65 \pm 17$ & $21 \pm 16$ & $70 \pm 21$ \\
\hline $\mathrm{N}-\mathrm{NH}_{4}{ }^{+} / \mathrm{NTK}$ & $0,6 \pm 0,1$ & $0,5 \pm 0,3$ & - \\
\hline $\mathrm{N}-\mathrm{NO}_{2}^{-}(\mathrm{mg} / \mathrm{L})$ & $0,04 \pm 0,06$ & $0,4 \pm 0,3$ & - \\
\hline $\mathrm{N}-\mathrm{NO}_{3}^{-}(\mathrm{mg} / \mathrm{L})$ & $0,10 \pm 0,23$ & $13 \pm 10$ & - \\
\hline NT & $86 \pm 26$ & $44 \pm 19$ & $50 \pm 20$ \\
\hline $\begin{array}{c}\text { Carga nitrogenada aplicada } \\
\left(\mathrm{Kg} . \mathrm{N} / \mathrm{m}^{3} . \mathrm{d}\right)\end{array}$ & \multicolumn{3}{|c|}{$0,151 \pm 0,066$} \\
\hline $\begin{array}{c}\text { Carga nitrogenada removida } \\
\left(\mathrm{Kg.N} / \mathrm{m}^{3} . \mathrm{d}\right)\end{array}$ & \multicolumn{3}{|c|}{$0,076 \pm 0,050$} \\
\hline
\end{tabular}

A relação Carbono/Nitrogênio (DQO/NTK) encontrada durante a operação do reator foi $4 \pm 2$. Reatores operados com baixa relação $\mathrm{C} / \mathrm{N}$ favorecem o desenvolvimento de organismos nitrificantes, contribuindo para um processo de nitrificação mais acentuado. Porém, em reatores operados com sistemas de aeração intermitente que favorecem o processo de NDS, a relação C/N necessária para que este processo ocorra com sucesso é superior ao desejado em reatores que visam apenas o processo de nitrificação, sendo estes valores maiores que $9[8,15]$.

Apesar da relação $\mathrm{C} / \mathrm{N}$ encontrada no presente trabalho ser considerada baixa para sistemas com NDS, com base nas concentrações de nitrogênio afluente e efluente, o percentual estimado de nitrificação foi $70 \pm 21 \%$ e de desnitrificação de $70 \pm 20 \%$. Assim constata-se que mesmo com a baixa relação $\mathrm{C} / \mathrm{N}$, observa-se que o processo de desnitrificação ocorreu com o mesmo potencial que o processo de nitrificação; sendo este fator possivelmente explicado pela coexistência de organismos nitrificantes e desnitrificantes no reator, principalmente aderidos ao material suporte.

\subsection{PERFIL DE REMOÇÃO DE MATÉRIA ORGÂNICA E NITROGÊNIO DURANTE OS 3 CICLOS DE AI}

Na Tabela 3 são apresentadas as médias de $\mathrm{pH}$, alcalinidade e $\mathrm{DQO}_{\mathrm{T}}$ efluente no $\mathrm{AE}$ e $\mathrm{AN}$ dos três ciclos analisados, durante $18 \mathrm{~h}$ de análise. Analisando esses resultados, nota-se que os valores de $\mathrm{pH}$ e alcalinidade obtidos nas fases $\mathrm{AE}$ e AN foram próximos. O mesmo pode ser observado nos valores de $\mathrm{DQO}_{\mathrm{T}}$ nas duas fases.

Tabela 3: Média dos parâmetros fisico-químicos $\left(\mathrm{pH}\right.$, alcalinidade $D Q O_{T}$, percentual de remoção de $\left.D Q O_{T}\right)$ analisados durante 18 horas de aeração intermitente.

\begin{tabular}{cccccc}
\hline FASE & $\mathbf{T ~}\left({ }^{\mathbf{0}} \mathbf{C}\right)$ & $\mathbf{p H}$ & $\begin{array}{c}\text { Alcalinidade } \\
\left(\mathbf{m g C a C O}_{3} / \mathbf{L}\right)\end{array}$ & $\begin{array}{c}\text { DQO } \\
(\mathbf{m g} / \mathbf{L})\end{array}$ & $\begin{array}{c}\text { \% Remoção } \\
\mathbf{D Q O}_{\mathbf{T}}\end{array}$ \\
\hline $\mathbf{A E}$ & $28 \pm 0,7$ & $8,8 \pm 0,1$ & $158 \pm 15$ & $49 \pm 8$ & $92 \pm 1$ \\
$\mathbf{A N}$ & $27 \pm 1,4$ & $8,7 \pm 0,1$ & $163 \pm 16$ & $47 \pm 2$ & $90 \pm 4$ \\
\hline
\end{tabular}

Os valores de $\mathrm{pH}$ não variaram consideravelmente durante as fases de AE/AN (Figura 2), mantendo-se dentro da faixa considerada como adequada para o processo de nitrificação e desnitrificação $(6,5-8)[16,17,18]$, porém os valores de alcalinidade variaram. 


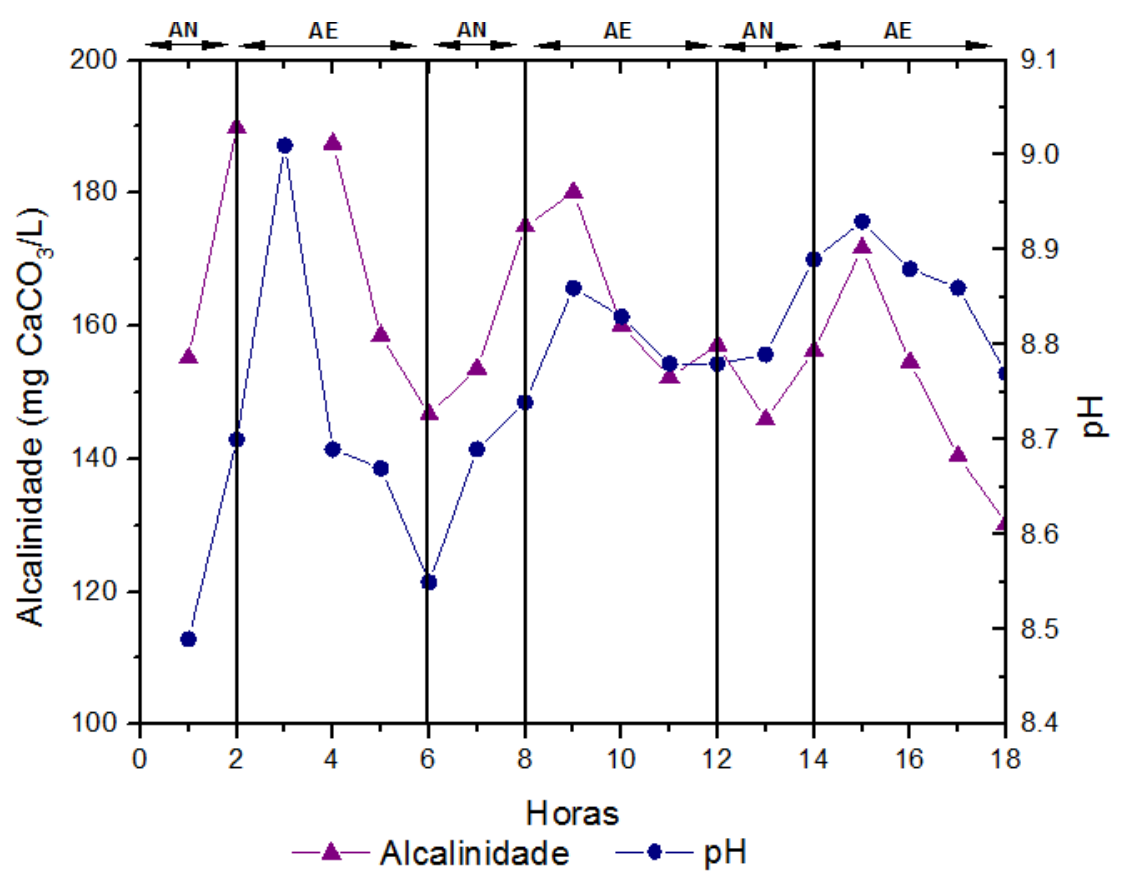

Figura 2: Dados de alcalinidade e pH durante o período de aeração intermitente analisado.

Para que o processo de nitrificação biológica ocorra é de fundamental importância a presença de alcalinidade no meio, visto que são consumidos cerca de $7,1 \mathrm{mg} \mathrm{CaCO}_{3}$ a cada mg de $\mathrm{N}$ amoniacal oxidado $[16,17]$ devido a liberação de $\mathrm{H}^{+}$no processo. A alcalinidade do meio na fase AE foi consumida, mas permaneceu acima de $120 \mathrm{mg} \mathrm{CaCO}_{3} / \mathrm{L}$. Segundo WEF, ASCE, EWRI (2005)[19] e USEPA (2008)[20], o mínimo de alcalinidade recomendado no efluente é de $50 \mathrm{mg}$ $\mathrm{CaCO}_{3} / \mathrm{L}$, embora seja preferível manter valores em torno de $100 \mathrm{mg} \mathrm{CaCO} / \mathrm{L}$.

Em relação às concentrações de DQO, observa-se que a concentração média efluente na fase AE $(49 \pm 8 \mathrm{mg} / \mathrm{L})$ e $\mathrm{AN}(47 \pm 2 \mathrm{mg} / \mathrm{L})$ foram próximos. Este resultado pode ser explicado possivelmente pelo fato dos organismos heterotróficos responsáveis pela remoção de matéria orgânica serem em sua grande maioria facultativos, podendo atuar em ambas as fases.

Analisando os resultados de $\mathrm{N}-\mathrm{NH}_{4}{ }^{+}$no efluente em ambas as fases, verifica-se que não houve um comportamento padrão da concentração deste no efluente, demostrando que a existência de $\mathrm{AE}$ e AN não influenciou de forma significativa os seus resultados (Tabela 4; Figura 3). Isto também pode ser constatado pelas médias de $\mathrm{N}-\mathrm{NH}_{4}{ }^{+}$, onde o percentual de remoção foi de $49 \pm 8 \%$ e $46 \pm 6 \%$ na fase AE e AN, respectivamente (Tabela 4). Na análise estatística aplicada para a comparação dos resultados de $\mathrm{DQO}_{\mathrm{T}}$ e $\mathrm{N}^{-\mathrm{NH}_{4}}{ }^{+}$, da fase $\mathrm{AE}$ e $\mathrm{AN}$, obteve-se que não houve diferença entre os valores encontrados $(\mathrm{p}>0,05)$. Em relação às concentrações de $\mathrm{N}_{-} \mathrm{NO}_{2}{ }^{-}$e N$\mathrm{NO}_{3}{ }^{-}$também não foram verificadas diferenças entre as concentrações efluentes entre as fases para os períodos com e sem aeração ( $p>0,05)$.

Tabela 4: Média dos parâmetros físico-químicos analisados (concentrações de $\mathrm{N}-\mathrm{NH}_{4}{ }^{+}, \mathrm{N}-\mathrm{NO}_{2}{ }^{-}, \mathrm{N}_{-} \mathrm{NO}_{3}{ }^{-}$, $\mathrm{OD}$, percentual de remoção de $\mathrm{NH}_{4}{ }^{+}$e desnitrificação) durante as 18 horas de aeração intermitente.

\begin{tabular}{|c|c|c|c|c|c|c|}
\hline Fase & $\begin{array}{c}\mathrm{N}-\mathrm{NH}_{4}{ }^{+} \\
(\mathrm{mg} / \mathrm{L})\end{array}$ & $\begin{array}{l}\mathrm{N}-\mathrm{NO}_{2}{ }^{-} \\
(\mathrm{mg} / \mathrm{L})\end{array}$ & $\begin{array}{l}\mathrm{N}-\mathrm{NO}_{3}{ }^{-} \\
(\mathrm{mg} / \mathrm{L})\end{array}$ & $\begin{array}{c}\text { OD } \\
(\mathrm{mg} / \mathrm{L})\end{array}$ & $\begin{array}{c}\% \\
\text { Remoção }^{\circ} \\
\mathrm{N}^{-\mathrm{NH}_{4}}{ }^{+} \\
\end{array}$ & $\begin{array}{c}\% \\
\text { Desnitrificação }\end{array}$ \\
\hline $\mathbf{A E}$ & $39 \pm 6$ & $1 \pm 0,1$ & $13 \pm 3$ & $4,1 \pm 0,6$ & $49 \pm 8$ & $62 \pm 10$ \\
\hline $\mathbf{A N}$ & $41 \pm 5$ & $1 \pm 0,1$ & $12 \pm 2$ & $1,3 \pm 1,4$ & $46 \pm 6$ & $61 \pm 9$ \\
\hline
\end{tabular}




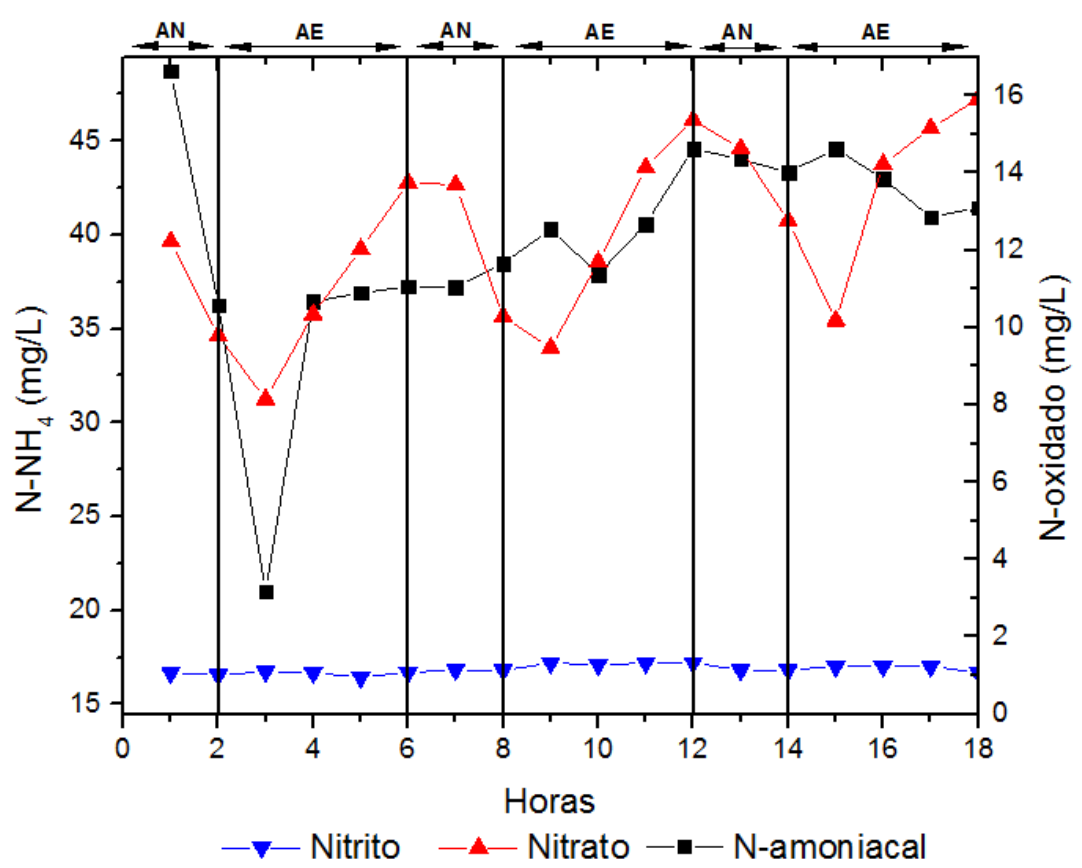

Figura 3: Dados de $\mathrm{N}-\mathrm{NH}_{4}{ }^{+}, \mathrm{N}-\mathrm{NO}_{2}{ }^{-}$e $\mathrm{N}-\mathrm{NO}_{3}{ }^{-}$durante o período de aeração intermitente analisado.

O fato de não ter sido encontrado diferenças em relação à concentração dos compostos nitrogenados analisados pode estar relacionado a alguns fatores, como a existência da NDS, devido à aeração intermitente e ao fato de o reator ser operado com recirculação interna; o que acaba favorecendo a diluição destes compostos no meio líquido e não sendo possível verificar sua variação [21]. O perfil de OD no meio durante os 3 ciclos analisados é apresentado na Figura 4. Constata-se que após o desligamento da aeração, o meio ainda apresentava concentrações de OD acima de $1 \mathrm{mg} / \mathrm{L}$ até 1 hora após o início da fase $\mathrm{AN}$, chegando a concentrações abaixo de 0,02 $\mathrm{mg} / \mathrm{L}$ de OD após este período. Este fato pode ter favorecido o processo de nitrificação no meio, mesmo quando a aeração estava desligada.

EPA (1993) [17] indica que o processo de nitrificação pode ser limitado em concentrações de OD de 0,5 - 2,5 mg/L. Eckenfelder (1989) [22] e WEF, ASCE, EWRI (2005)[19] recomendam que o nível de OD seja superior a 2,0 mg/L, porém Stenstrom e Poduska (1980)[23] reportam que o processo de nitrificação pode ocorrer em níveis de OD de 0,3 a 4,0 mg/L.

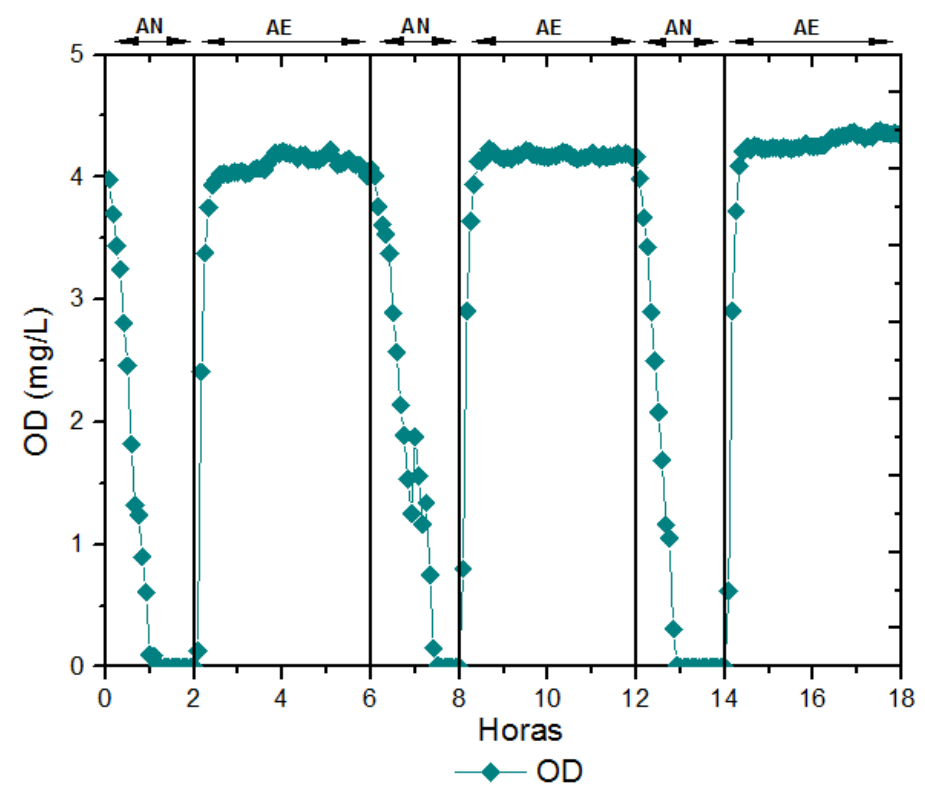

Figura 4: Concentração de OD duração os ciclos de AI analisados. 
Durante o período em que a concentração de OD foi menor que $0,02 \mathrm{mg} / \mathrm{L}$, a alcalinidade do meio foi recuperada; indicando que ocorreu o processo de desnitrificação que foi confirmado pela diminuição de $\mathrm{NO}_{3}{ }^{-}$no efluente. $\mathrm{Na}$ desnitrificação, a alcalinidade do meio é recuperada com produção teórica de cerca de $3,57 \mathrm{mg}$ de alcalinidade $\mathrm{CaCO}_{3}$ por $\mathrm{mg}$ de $\mathrm{N}_{-}-\mathrm{NO}_{3}{ }^{-}$reduzido a $\mathrm{N}_{2}$ [17].

Mesmo em concentrações de OD acima de $1 \mathrm{mg} / \mathrm{L}$, pode-se constatar que o processo de desnitrificação ocorreu, pois a alcalinidade do meio ainda continuou a aumentar como pode ser observado na Figura 3. Este fato pode ser explicado pela existência do biofilme no material suporte que viabiliza a ocorrência de organismos desnitrificantes em camadas inferiores deste que, mesmo quando a concentração de OD no meio líquido já encontra-se elevada, favorece a formação de ambientes anóxicos nas camadas internas do biofilme, pois o oxigênio não difunde e possibilita que o processo de desnitrificação continue ocorrendo eficientemente.

Alguns trabalhos que utilizam material suporte em sistemas biológicos que visam a remoção de nitrogênio relatam a redução de nitrato em concentrações de OD superiores a $4 \mathrm{mg} / \mathrm{L}$, relacionando a viabilidade da desnitrificação nestas concentrações de OD devido a existência de gradientes de concentração de substratos no biofilme formado no material suporte ou em flocos $[4,8,24,25]$.

Apesar de os resultados de alcalinidade e $\mathrm{NO}_{3}{ }^{-}$indicarem que o processo de desnitrificação foi mais acentuado durante a fase $\mathrm{AN}$, os valores médios de desnitrificação em ambas as fases foram praticamente iguais, com médias de $62 \pm 10 \%$ na fase $\mathrm{AE}$ e $61 \pm 9 \%$ na fase $\mathrm{AN}$. Este fato pode ser explicado pela possível existência de zonas anóxicas em regiões profundas do material suporte utilizado, em virtude da dificuldade de penetração do OD devido a formação do biofilme [26, 27].

Santos (2014) [28] realizando um estudo da concentração de OD no interior do biofilme formado em espuma de poliuretano presente em reator de leito estruturado tratando esgoto sintético, em fluxo contínuo e aeração intermitente, constatou em um biofilme com espessura de aproximadamente $1570 \mu \mathrm{m}$, uma zona aeróbia de $670 \mu \mathrm{m}$ e uma zona anóxica de $900 \mu \mathrm{m}$. Esta estratificação favorece o processo de NDS, fazendo com que o nitrogênio oxidado em zonas profundas possa ser consumido e transformado em nitrogênio gasoso; o que faz com que o reator não apresente picos de concentração de nitrogênio oxidado no efluente.

\section{CONCLUSÃO}

De maneira geral, o reator operado sobre as condições trabalhadas (TDH $16 \mathrm{~h}$, ciclos de aeração e não aeração de $4 \mathrm{~h} / 2 \mathrm{~h}$, respectivamente com recirculação do efluente) pode ser considerado como uma alternativa para o tratamento de esgoto sanitário com vistas à remoção simultânea de matéria orgânica e nitrogênio, obtendo percentuais consideráveis de remoção de $\mathrm{DQO}_{\mathrm{T}}(88 \pm 4 \%)$ e NTK $(72 \pm 6 \%)$.

Os resultados alcançados durante as $18 \mathrm{~h}$ de análise permitem concluir que, mesmo o sistema operando sob condições de aeração e não aeração, não pode ser constatada diferenças (picos) em relação às concentrações de matéria orgânica e nitrogênio oxidado no efluente. Da análise estatística aplicada aos dados de $\mathrm{DQO}_{\mathrm{T}}, \mathrm{NH}_{4}{ }^{+}, \mathrm{NO}_{2}{ }^{-}$e $\mathrm{NO}_{3}{ }^{-}$obteve-se que não houve diferença significativa entre estes parâmetros durante a fase $\mathrm{AE}$ e $\mathrm{AN}$ no reator durante o período analisado. Estes resultados podem ser explicados principalmente pelo possível processo de NDS e pela recirculação do efluente

Dos resultados de alcalinidade no meio líquido e OD aponta-se que o processo de desnitrificação ocorreu mesmo em concentração de OD acima de $1 \mathrm{mg} / \mathrm{L}$. Este fato pode ter ocorrido possivelmente em decorrência da formação de biofilme no material suporte, o que possibilita a existência de gradientes de OD, formando zonas anóxicas no interior do biofilme. 


\section{AGRADECIMENTOS}

Este trabalho foi financiado pela Companhia de Saneamento do Paraná (SANEPAR/Paraná/Fundação Araucária) e Coordenação de Aperfeiçoamento de Pessoal de Nível Superior (CAPES).

1. Bhuvanesh S, Maneesh N, Sreekrishnan T. R. Start-up and performance of a hybrid anoxic reactor for biological denitrification. Bioresource Technology. 2013; 129:74-84, doi: 10.1016/j.biortech.2012.

2. Pochana K, Keller J. Study of factors affecting simultaneous nitrification and denitrification (SND). Water Sci. Technol. 1999; 39(6):61-68, doi:10.1016/S0273-1223(99)00123-7.

3. Wang J, Peng Y, Wang S, Gao Y. Nitrogen Removal by Simultaneous Nitrification and Denitrification via Nitrite in a Sequence Hybrid Biological Reactor. Chinese Journal of Chemical Engineering. 2008 Oct; 16(5):778-784, doi:10.1016/S1004-9541(08)60155-X.

4. Virdis B, Read ST, Rabaey K, Rozendal RA, Yuan Z, Keller J. Biofilm stratification during simultaneous nitrification and denitrification (SND) at a biocathode. Bioresource Technology. 2011 Jan; 102(1):334-41, doi: 10.1016/j.biortech.2010.06.155.

5. Guo J, Zhang L, Cheng W, Ma F, Liu H, Tian Y. The regulation and control strategies of a sequencing batch reactor for simultaneous nitrification and denitrification at different temperatures. Bioresour Technol. 2013 Apr; 133:59-67, doi:10.1016/j.biortech.2013.01.026.

6. Capodici M, Di Bella G, Di Trapani D, Torregrossa M. Pilot scale experiment with MBR operated in intermittent aeration condition: Analysis of biological performance. Bioresour Technol. 2015 Feb; 177:398-405, doi: 10.1016/j.biortech.2014.11.075.

7. Collivignarelli C, Bertanza G. Simultaneous nitrification denitri- fication processes in activated sludge plants: performance and applicability. Water Science and Tecnology. 1999; 40(4-5):187-194, doi:10.1016/S0273-1223(99)00575-2.

8. Moura RB, Damianovic MHRZ, Foresti E. Nitrogen and carbon removal from synthetic wastewater in a vertical structured-bed reactor under intermittent aeration. J. Environ. Manage. 2012 May; 98:163167, doi:10.1016/j.jenvman.2012.01.009.

9. Chang YJ, Tseng SK. A novel double-membrane system for simultaneous nitrification and denitrification in a single tank. Lett Appl Microbiol. 1999 Jun; 28(6):453-6.

10. Turk O, Mavinic DS. Benefits of using selective-inhibition to remove nitrogen from highly nitrogenous wastes. Environ. Technol. Lett. 1987 Dec; 8:419-426, doi:10.1080/09593338709384500.

11. American Public Health Association (APHA). Standards Methods for the Examination of Water e Wastewater. Washington, D.C; 2005.

12. Von Sperling M. Introdução a qualidade das águas e tratamento de esgoto. Departamento de Engenharia Sanitária da Universidade Federal de Minas Gerais. Belo Horizonte; 2005.

13. Jordão EP, Pessoa CA. Tratamento de esgoto doméstico. Rio de Janeiro: Segrac; 2005.

14. Barana AC, Lopes DD, Martins TH, Pozzi E, Damianovic MHRZ, Del Nery V, Foresti, E. Nitrogen and organic matter removal in an intermittently aerated fixed-bed reactor for post-treatment of anaerobic effluent from a slaughterhouse wastewater treatment plant. Journal of Environmental Chemical Engineering. 2013 Sep; 1(3):453-459, doi:10.1016/j.jece.2013.06.015.

15. Fu B, Liao X, Ding L, Ren H. Characterization of microbial community in an aerobic moving bed biofilm reactor applied for simultaneous nitrification and denitrification. World J. Microbiol. Biotechnol, 2010 Nov; 26(11):1981-1990, doi: 10.1007/s11274-010-0382-y.

16. Sedlak RL. Phosphorus and Nitrogen Removal From Municipal Wastewater. United States; 1991.

17. Environmental Protection Agency (EPA). Manual: Nitrogen. Cincinnati: Ohio; 1993.

18. Mayer M, Smeets W, Braun RE, Fuchs W. Enhanced ammonium removal from liquid anaerobic digestion residuals in an advanced sequencing batch reactor system. Water Sci Technol. 2009; 60(7):1649-60, doi: 10.2166/wst.2009.546.

19. WEF, ASCE, EWRI. Biological nutrient removal (BNR) operation in wastewater treatment plants. New York; 2005.

20. United States Environmental Protection Agency (USEPA). Municipal nutrient removal technologies Reference document. EPA/832/R-08/006; 2008.

21. Moura RB. Remoção de material orgânica e nitrogênio de esgoto sanitário em reator de leito estruturado submetida a aeração intermitente e recirculação do efluente. [Tese]. São Carlos: Escola de Engenharia de São Carlos; 2014. 132p.

22. Eckenfelder WW. Industrial water pollution control. McGraw-Hill, Singapura; 1989. 
23. Stenstrom MK, Poduska RA. The effect of dissolved oxygen concentration on nitrification. Water Research. 1980; 14(6):643-649, doi:10.1016/0043-1354(80)90122-0.

24. Guo J, Zhang L, Cheng W, Ma F, Liu H, Tian Y. The regulation and control strategies of a sequencing batch reactor for simultaneous nitrification and denitrification at different temperatures. Bioresour Technol. 2013; 133:59-67, doi:10.1016/j.biortech.2013.01.026.

25. Yilmaz G, Lemair, R, Keller J, Yuan Z. Simultaneous nitrification, denitrification, and phosphorus removal from nutrient-rich industrial wastewater using granular sludge. Biotechnology and Bioengineering. 2008; 100(3):529-541.

26. Luciano A, Viotti P, Mancini G, Torretta V. An integrated wastewater treatment system using a BAS reactor with biomass attached to tubular supports. J. Environ. Manage. 2012 Dec; 113:51-60, doi:10.1016/j.jenvman.2012.08.034.

27. Daniel LMC, Pozzi E, Foresti E, Chinalia FA. Removal of ammonium via simultaneous nitrificationedenitrification nitrite-shortcut in a single packed-bed batch reactor. Bioresource Technology. 2009; 100(30): 1100-1107.

28. Santos CED. Influencia da relação carbono/nitrogênio e da fonte de carbono no processo de nitrificação e desnitrificação simultânea em um reator de leito estruturado. [Dissertação]. São Carlos: Escola de engenharia de São Carlos; 2014. 144p. 